\title{
Advances in computational and mathematical chemistry
}

\author{
Jesús Vigo-Aguiar • Juan L. G. Guirao
}

Received: 24 December 2010 / Accepted: 9 March 2011 / Published online: 18 March 2011

(C) Springer Science+Business Media, LLC 2011

From June 26 to 30, 2010, the "10th International Conference on Computational and Mathematical Methods in Science and Engineering” (CMMSE 2010) was celebrated in Almería (Spain), where two hundred experts coming from various countries discussed the latest developments and techniques for solving problems coming from the sciences and engineering, using, as a common vehicle, mathematics in general, and specifically, mathematics as helped by computation.

The logistics of this conference followed a division of the topics into scientific sessions. Specifically, one of the sessions organized was devoted to "Mathematical Chemistry" or "Mathematical/Computer Chemistry". In this session, many different problems were presented and new tools were introduced to the scientific community. It appears certain, that these tools will be used in the future for solving additional problems which are still considered open problems at this moment.

As a consequence of the importance of this session within the framework of the CMMSE 2010 conference, the Journal of Mathematical Chemistry publishes this special issue with the aim of providing a widely accessible forum to the best relevant contributions presented at CMMSE 2010.

The present issue is composed by six research papers which give an overview of the state of the art of the approaches to specific problems discussed by the participants at the session.

This is one of several papers published in Journal of Mathematical Chemistry, "Special Issue: CMMSE 2010", with invited editorial contribution by Prof. Jesus Vigo-Aguiar.

J. Vigo-Aguiar

Universidad de Salamanca, Salamanca, Spain

J. L. G. Guirao ( $\varangle)$

Universdad Politécnica de Cartagena, Cartagena, Spain

e-mail: juan.garcia@upct.es 
The first contribution, entitled "Optimal Cooling Strategies in Polymer Crystallization" is presented by Ramón Escobedo and Luis A. Fernández, where an optimal control problem for cooling strategies in polymer crystallization processes is described by a deterministic model and the problem is solved within the framework of a free boundary problem. The second paper, "Modeling of neutron activation process with Americium Beryllium source. Application to the activation of fluorspar samples" by M.A. Rey-Ronco, T. Alonso-Sánchez and M. P. Castro-García is an innovative work where the mathematical models representing the phenomena occurring in a neutron activation process are presented. In the third paper of this special issue, with the interrogative title "Is there anything left to say on enzyme kinetic constants and quasi-steady state approximation?", Alberto Maria Bersani and Guido Dell' Acqua re-examine the commonly accepted meaning of the two kinetic constants characterizing any enzymatic reaction, according to Michaelis-Menten kinetics. By expanding the solutions of the ODEs governing the reaction in terms of exponentials, the authors determine a new constant, and correct some misinterpretations of the current biochemical literature. The next paper, entitled "An efficient and scalable block parallel algorithm of Neville Elimination as a tool for the CMB maps problem" is a joint work of P. Alonso, R. Cortina, J. Ranilla and A.M. Vidal, where the performance of several versions of a block parallel algorithm is analyzed with the aim of applying Neville elimination in a distributed memory parallel computer. The following paper presents the work of Raquel Cumeras et al., with the title "Modelling a P-FAIMS with Multiphysics FEM", with the aim of modeling different kinds of ions within a P-FAIMS approach, considering the main factors involved in their motion inside the drift tube. Finally, Juan L.G. Guirao and T. de Bustos present a paper entitled "Dynamics of pseudo-radioactive chemical products via sampling theory", where an alternative proof of the Shannon's sampling theorem is presented for non band limited signals. This result is applied for studying the dynamics of pseudo-radioactive chemical products.

In the opinion of the Guest Editors, this collection of papers summarize the spirit of the collection of results presented in the special session devoted to Mathematical Chemistry at CMMSE 2010. We also hope that these works inspire future research in this field. 